\title{
REYNOLDS, MAXWELL AND THE RADIOMETER, REVISITED
}

\author{
Holger Martin \\ Thermische Verfahrenstechnik, Karlsruhe Institute of Technology (KIT) \\ Karlsruhe, Germany
}

\section{ABSTRACT}

In 1969, S. G. Brush and C. W. F. Everitt published a historical review, that was reprinted as subchapter 5.5 Maxwell, Osborne Reynolds, and the radiometer, in Stephen G. Brush's famous book The Kind of Motion We Call Heat. This review covers the history of the explanation of the forces acting on the vanes of Crookes radiometer up to the end of the $19^{\text {th }}$ century. The forces moving the vanes in Crookes radiometer (which are not due to radiation pressure, as initially believed by Crookes and Maxwell) have been recognized as thermal effects of the remaining gas by Reynolds - from his experimental and theoretical work on Thermal Transpiration and Impulsion, in 1879 - and by the development of the differential equations describing Thermal Creeping Flow, induced by tangential stresses due to a temperature gradient on a solid surface by Maxwell, earlier in the same year, 1879.

These fundamental physical laws have not yet made their way into the majority of textbooks of heat transfer and fluid mechanics so far.

A literature research about the terms of Thermal Transpiration and Thermal Creeping Flow, in connection with the radiometer forces, resulted in a large number of interesting papers; not only the original ones as mentioned in subchapter 5.5 of Brush's book, but many more in the earlier twentieth century, by Martin Knudsen, Wilhelm Westphal, Albert Einstein, Theodor Sexl, Paul Epstein and others.

The forces as calculated from free molecular flow (by Knudsen), increase linearly with pressure, while the forces from Maxwell's Thermal Creeping Flow decrease with pressure. In an intermediate range of pressures, depending on the characteristic geometrical dimensions of flow channels or radiometer vanes, an appropriate interpolation between these two kinds of forces, as suggested by Wilhelm Westphal and later by G. Hettner, goes through a maximum. Albert Einstein's approximate solution of the problem happens to give the order of magnitude of the forces in the maximum range.

A comprehensive formula and a graph of the these forces versus pressure combines all the relevant theories by Knudsen (1910), Einstein (1924), Maxwell (1879) (and Hettner (1926), Sexl (1928), and Epstein (1929) who found mathematical solutions for Maxwells creeping flow equations for nonisothermal spheres and circular discs, which are important for thermophoresis and for the radiometer).

The mechanism of Thermal Creeping Flow will become of increasing interest in micro- and submicro-channels in various new applications, so it ought to be known to every graduate student of heat transfer in the future.

That's one of the reasons why some authors have recently questioned the validity of the classical Navier-Stokes, Fourier, and Fick equations: Dieter Straub (1996) published a book on an Alternative Mathematical Theory of Non-equilibrium Phenomena. Howard Brenner (since 2005) wrote a number of papers, like Navier-Stokes, revisited, and Bi-velocity hydrodynamics, explicitly pointing to the forces acting on the vanes of the lightmill, to thermophoresis and related phenomena. Franz Durst (since 2006) also developed modifications of the classical Navier-Stokes equations. So, Reynolds, Maxwell, and the radiometer may finally have initiated a revision of the fundamental equations of thermofluiddynamics and heat- and mass transfer.

\section{INTRODUCTION}

The history of the theoretical explanations of the forces acting on the vanes of Crookes's radiometer (and the related problems of Thermal Transpiration and Thermophoresis) can 
now be subdivided into three separate periods. Each of these periods had a duration of about 20 to 25 years:

1873 - 1897: The period starting with Crookes's discovery of the existence of a radiometric force. Important papers were written by Crookes (1875 - 1877), by Reynolds (1876 - 1879), and by Maxwell (1879). This first period is very well covered by chapter 5.5 of Brush's book on the history of the kinetic theory of gases during the $19^{\text {th }}$ century. About 20 authors published at least 30 papers on the topic from 1874 to 1897.

1909 - 1930: This second period of research is characterized by Knudsen's work (1909-1930), by the experimental work of Westphal (1910-19..) and Hettler (1924-1928), as well as by theoretical work by Einstein (1924), Hettler (19261928), Epstein (1927), and Sexl (1928).

1980 - 2010: New interest in the problem came up for several reasons after a period of about 50 years of relative scarce interest in the late $20^{\text {th }}$ and the beginning $21^{\text {st }}$ centuries.

Microchannel applications made transitional effects, belonging to Knudsen numbers of the order of one more important. That's why new patents on so-called Knudsen pumps, based on thermal transpiration, and, consequently, modifications of the Navier-Stokes, Fourier, and Fick equations were proposed since 1996 by Straub, since 2005 by Brenner, and since 2006 by Durst.

\begin{tabular}{|c|c|c|}
\hline \multicolumn{3}{|c|}{ NOMENCLATURE } \\
\hline$d$ & $\mathrm{~m}$ & diameter (tube, disc) \\
\hline$F$ & $\mathrm{~N}$ & force \\
\hline$L$ & $\mathrm{~m}$ & length, $L^{2}=(\pi / 4) d^{2}$ area \\
\hline$\tilde{M}$ & $\mathrm{~kg} \mathrm{kmol}^{-1}$ & molar mass \\
\hline$\dot{n}$ & $\mathrm{kmol} \mathrm{m}^{-2} \mathrm{~s}^{-1}$ & molar flux \\
\hline$p$ & $\mathrm{~Pa}$ & pressure \\
\hline$r$ & $\mathrm{~m}$ & radius \\
\hline$R$ & $\mathrm{~J} \mathrm{~kg}^{-1} \mathrm{~K}^{-1}$ & gas constant $(=\tilde{R} / \tilde{M})$ \\
\hline$\widetilde{R}$ & $\mathrm{~J} \mathrm{kmol}^{-1} \mathrm{~K}^{-1}$ & universal gas constant \\
\hline$t$ & S & time \\
\hline$T$ & $\mathrm{~K}$ & temperature \\
\hline$u$ & $\mathrm{~m} \mathrm{~s}^{-1}$ & velocity in $x$-direction \\
\hline$v$ & $\mathrm{~m} \mathrm{~s}^{-1}$ & velocity in $y$-direction \\
\hline$w$ & $\mathrm{~m} \mathrm{~s}^{-1}$ & velocity in $\mathrm{z}$-direction \\
\hline$\Delta$ & 1 & difference \\
\hline$\Lambda$ & $\mathrm{m}$ & mean free path \\
\hline$\rho$ & $\mathrm{kg} \mathrm{m}^{-3}$ & density \\
\hline$\eta$ & Pas & viscosity \\
\hline
\end{tabular}

\section{CROOKES, REYNOLDS, MAXWELL}

The starting point of the whole story may be seen in the discovery of a new kind of forces observed by the chemist William Crookes, around 1873, in his private laboratory in London. In 1861, he had discovered a new element, thallium, by using the spectral analysis, developed by the chemist Bunsen and the physicist Kirchhoff in 1859 at Heidelberg. In
1860, and 1861, these German scientists had already discovered the elements cesium and rubidium by their new method. Crookes tried to find out the molecular mass of his new element by a series of careful weighings, partly under vacuum. During these experiments he observed that a hot body in the vicinity of a mass on the balance can change the weight of that mass! When put below the mass on the balance the weight decreased, while it increased when the hot body was put above it. His literature studies showed him that Fresnel had found this same effect already in 1825. But when Crookes observed it, around 1873, James Clerk Maxwell had already postulated, and theoretically predicted the existence of a pressure exerted by radiation. Crookes built several radiometers, typically with four circular (or quadratic) discs as vanes mounted on a vertical axis which could rotate around this axis on a needle in an evacuated glass bulb. The vanes were made from pith (and other materials), blackened by soot on one side and reflecting on the other side (pith is white by nature).

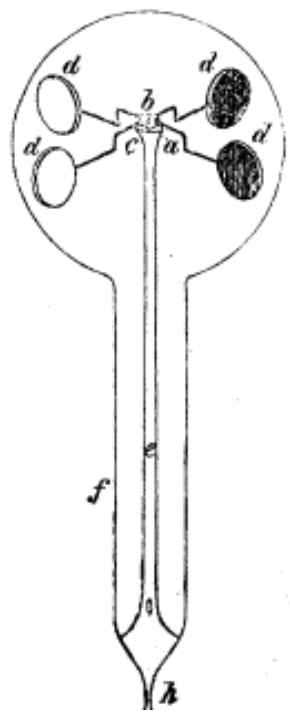

Figure 1 Crookes radiometer

The glass bulb was evacuated as good as possible at the time, and then sealed. Sunlight, or the light from a candle, or a number of candles, caused the vanes to rotate quickly around their axis, which was demonstrated by Crookes at a meeting of the Royal Society. This demonstration of a surprising force caused a hype of interest in the radiometer problem, which led to a great number of papers published on this topic within the next few years. This first part of the story is well documented by subchapter 5.5 of $S$. G. Brush's book The Kind of Motion We Call Heat ${ }^{1}$. Crookes's original idea, namely that the radiometer were rotating because of a direct effect of radiation pressure, was soon shown to be wrong by other scientists.

At least three reasons were found why it couldn't be the direct effect of radiation: If it where the light pressure the rotation must have been with the reflecting surfaces being pushed away from the radiation, which is just opposite to the observed motion. The order of magnitude of the light pressure (as calculated from Maxwell's theory) was much lower than the forces observed. And finally, if it were the direct effect of radiation, the bulb, when suspended on a thin wire must show a tendency to rotate in the same direction as the vanes (due to the friction of the needle bearing). In 1876, Arthur Schuster, a younger colleague of Reynolds at Owens College in Manchester showed that the bulb rotates in the direction opposite to the vanes. From this experimental result it was easy to conclude that the remaining gas in the (partly) evacuated bulb must play a crucial role in the motion of the vanes. 
Osborne Reynolds, the first professor of engineering at Owens College in Manchester, started a fundamental research program to find out the nature of the forces acting on the vanes of the lightmill (Crookes's radiometer). Schuster's and Reynolds's papers about their research concerning the radiometer were published in 1876. A number of other authors discussed the topic in the period from 1874 to 1897 (see Brush ${ }^{1}$ ). Reynolds tried to solve the problem by careful experiments. He studied a related process, which he called Thermal Transpiration, that is the flow of a single gas (air or hydrogen), through a porous slab (made of stucco, or meerschaum) driven by a temperature gradient. The chambers on both sides of the slab were kept at fixed temperatures (with a difference of typically $160^{\circ} \mathrm{F}=88.9$ $\mathrm{K}$ ); the total pressure was varied from atmospheric pressure down to less than $1 / 100$ of an atmosphere, and the steady state pressure difference between the hotter and the colder side varied from about $3 / 100$ to $3 / 10,000$ of an atmosphere. In steady state the molar fluxes (see eqn. 1) from cold (subscript $i$ $=c$ ) to hot $(i=h)$ - initially (when the pressure on both sides is still equal) greater than in the other direction - and from hot to cold have become equal, while a certain pressure difference from hot to cold has built up. In the low-pressure limit, the molar fluxes can be obtained from the product of molar density, $\tilde{\rho}=\rho / \tilde{M}=p /(\tilde{R} T)$, and mean molecular velocity, $\bar{w}$ :

$$
\dot{n}_{i}=\frac{1}{4}(\tilde{\rho} \bar{w})_{i} \quad i=h, c
$$

Inserting the dependencies on pressure, $p$, and temperature, $T$, of both quantities results in:

$$
\dot{n}_{i}=\frac{1}{\sqrt{2 \pi \tilde{M} \tilde{R}}}\left(\frac{p}{\sqrt{T}}\right)_{i} \quad i=h, c
$$

With the two fluxes being equal and opposite (that is, there is no net flux) in steady state, the pressure difference becomes:

$$
\Delta p_{\text {steadystate }} \approx p \frac{\Delta T}{2 T} \quad p<<p_{\text {opt }}
$$

This was first found by Osborne Reynolds ${ }^{2}$ in 1879. The pressure difference, for very low pressures, increases linearly with the total pressure.

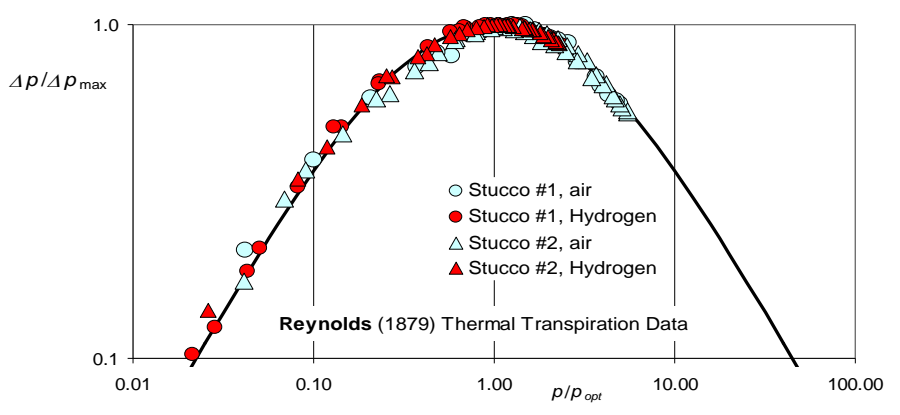

Figure 2 Reynolds's thermal transpiration data replotted as relative pressure difference vs. pressure ratio (log-log-plot). Curve from eq. (7) with $n=0.44$.
For higher pressures, however, this simple law, derived from the kinetic theory, doesn't apply any more. Reynolds's thermal transpiration experiments show that the pressure difference goes through a maximum and tends to decrease inversely with pressure for higher pressures. Figure 2 shows Reynolds's data for stucco slabs with two different pore sizes and experiments with air and hydrogen replotted as $\Delta p / p_{\max }$ versus $p / p_{\text {opt }}$, where the reference values, $p_{\max }$, and, $p_{\text {opt }}$, were fitted to bring the data together on a single curve (Reynolds's data for meerschaum slabs, not shown in this figure, have their optimum pressure at about 30 inches $\mathrm{Hg}$ (atmospheric pressure), so they do not show the descending branch). Reynolds had chosen the thermal transpiration experiments because he recognized that the light mill in principle could work at higher gas pressures if the vanes were accordingly reduced in size. The mean free path of the gas molecules (inversely proportional to the pressure, $p$ ) as the scale for the appropriate size of the vanes led him to the conclusion that a lightmill ought to have extremely small vanes to work at atmospheric pressures. The impossibility to build such a micro- or rather nano-scaled lightmill made him think of a simpler alternative. Instead of moving vanes, fixed walls of a porous slab would cause the gas to move (from cold to hot) if a temperature gradient was maintained across their width.

Reynolds's very detailed report about his experiments on both thermal transpiration and impulsion (that is the force acting on the radiometer vanes) happened to be reviewed by Maxwell (see Brush ${ }^{1}$ ). Maxwell had himself written a theoretical paper On Stresses in Rarified Gases arising from Inequalities in Temperature ${ }^{3}$, but this didn't lead him to a reasonable explanation of the nature of the forces acting on the vanes of the radiometer. After reading Reynolds's manuscript, however, he checked some new theoretical ideas regarding a tangential thermal creeping flow induced by temperature gradients along the surface of a plane wall. Initially he had only regarded normal stresses, which depend on second spatial derivatives of temperature and therefore would not cause a steady-state motion of the vanes in a continuous gas. The new idea of a (tangential) slip flow of rarefied gases was based on some earlier discoveries of slip phenomena by German scientists. So he developed a theory of thermal creeping flow (from colder to hotter regions on a surface) in an annex to his paper, where he quoted the unpublished(!) paper of Reynolds, crediting him to have discovered the effect, but critisizing his methods. Maxwell's paper was published in 1879 (and unfortunately he died in november 5 , the same year). Reynold's paper was published later in the same year 1879, and, quite under-standably, he was upset by Maxwell's behavior (see Brush $^{1}$ for more details of this fascinating story). Maxwell's theory in fact gives an explanation for the descending branch of the curve in Figure 2.

$$
\Delta p_{\text {steady state }}=\frac{p_{c h}^{2}}{p} \frac{\Delta T}{T} \quad p>>p_{\text {opt }}
$$

In eq. (3) the influence of temperatures (for $\Delta T<<T$ ) was linearized, to show the same dependency as in eq. (4). The 
characteristic pressure, $p_{\mathrm{ch}}$, in equation (4) is a function of gas properties (viscosity and molar mass), the characteristic diameter, $d$, of the capillaries of the porous body, and of temperature, $T$ (with $\bar{w}=[8 \tilde{R} T /(\pi \tilde{M})]^{1 / 2}$ as before).

$$
p_{c h}=(3 \pi)^{1 / 2} \eta \frac{\bar{w}}{d}
$$

So in principle, the problem of explaining the nature of the forces acting on Crookes radiometer was solved (at least qualitatively) by the work of Reynolds and Maxwell in 1879.

\section{KNUDSEN, WESTPHAL, EINSTEIN}

The scientific interest in the problem accordingly ebbed away and nearly no more papers were published about this topic after 1897. Brush's ${ }^{1}$ report about this scientific episode ends here. About thirty years after Reynolds ${ }^{2}$ and Maxwell $^{3}$ however, Martin Knudsen ${ }^{4-9}$, a young professor at the University of Kopenhagen started a series of investigations on rarefied gases. What we are now familiar with as the Knudsen number, the ratio of the mean free path of the gas molecules to a characteristic dimension of the flow passage, was nothing else than what Osborne Reynolds had postulated 30 years earlier in his voluminous and, obviously, not very well known paper $^{2}$ (125 pp.). Knudsen partly re-discovered what Reynolds had called thermal transpiration. Later, one of his colleagues, a certain Prof. K. Prytz, seems to have pointed him to Reynolds's $\operatorname{paper}^{1}$ (see Knudsen ${ }^{6}$, pp. 639-640). For low pressures his approximate solution for the radiometric force is (see eq. (3)):

$$
F_{\text {Knudsen }} \cong p L^{2} \frac{\Delta T}{4 T} \quad p<<p_{\text {opt }}
$$

Ten years later the German physicist Wilhelm Westphal ${ }^{10}$ undertook a rigorous experimental investigation on the forces acting on the vanes of the radiometer. He found out that a plot of the forces acting on the vanes versus the logarithm of the absolute pressure usually shows a curve which is symmetrical to a vertical line through the (optimal) pressure at the maximum of the force (see Fig. 3). It is interesting to find that Reynolds's thermal transpiration data (in Figure 2) show the same behavior.

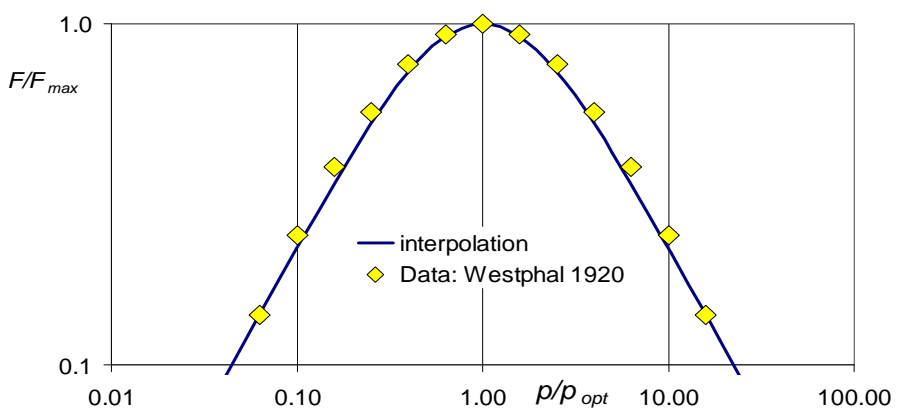

Figure 3 Westphal's data on radiometer forces vs. pressure. Interpolation from equation (7) with $n=0.85$.
The interpolation curves in Figures 2 and 3 were calculated from the same empirical function, combining the two different laws (increasing with $p$, and decreasing with $1 / p$ ):

$$
F / F_{\max }=\left(\frac{2}{\left(p / p_{\text {opt }}\right)^{n}+\left(p_{\text {opt }} / p\right)^{n}}\right)^{1 / n}
$$

The exponent $n$ was fitted for the thermal transpiration data (Fig. 2) to a value of $n_{\text {Reynolds }}=0.44$, and for the radiometer data (Fig. 3) to $n_{\text {Westphal }}=0.85$, respectively. The symbols in Figure 3 are averaged values from 42 series of experiments with 15 different radiometers from Table 2 in the paper by Wilhelm $H$. Westphal ${ }^{10}$ (part II) p. 435.

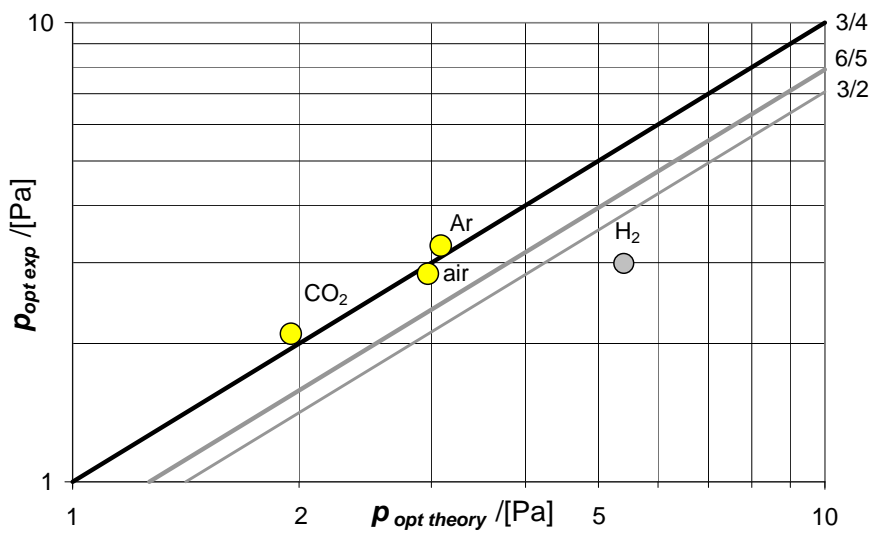

Figure 4 Optimal pressures from Westphal's experiments with different gases. Numerical values: $C_{\mathrm{s}}=3 / 4\left(\right.$ Maxwell $\left.^{3}\right),=3 / 2$ $\left(B r e n n e r^{16}\right)$. Lines from eqation (8) (as calculated from the thermal creeping flow solutions of Epstein ${ }^{13}$ and Sexl ${ }^{14}$ )

$$
p_{\text {opt }}=\left(16 \pi C_{s}\right)^{1 / 2} \frac{\eta}{L} \sqrt{\frac{\tilde{R} T}{\tilde{M}}}
$$

Though the data (excluding hydrogen) seem to fit better with Maxwell's value of the constant $C_{\mathrm{s}}$, the higher (present day) value $C_{\mathrm{s}}=3 / 2$ ought to be used for the sake of consistency.

In 1924, even Albert Einstein ${ }^{11}$ published a paper 'On the Theory of the Radiometric Forces'. He argued that a small particle or a radiometer vane, even if its length scale, $L$, is greater than the mean free path of the gas molecules, will feel a pressure difference due to a temperature gradient in a small zone around the edges of the vane. He found that the force can be calculated from an approximate formula (which turns out to be very close to Knudsen's equation (6)), if the vane area, $L^{2}$, is replaced by the area of a small strip around the edges, $4 L \Lambda$, of the width of a mean free path, $\Lambda$ :

$$
F_{\text {Einstein }}=p L \Lambda \frac{\Delta T}{T}
$$

Though equation (9), resulting from Einstein's ${ }^{11}$ order-ofmagnitude formula (see his equation 11a, on p. 5, where $L$ ought to be taken as the total length of the edges, i. e., $4 L$ ) may 
be 'wrong' by a factor of $1 / 4$, it seems to be much more consistent with Knudsen's result (6).

Maxwell's ${ }^{3}$ thermal creeping flow equations were solved for spherical particles in a linear temperature gradient, and for a given surface temperature distribution (due to radiation) in an isothermal gas were solved in the years from 1926 to 1929 by Hettner $^{12}$, by Sexl ${ }^{13}$, and by Epstein ${ }^{14}$. Epstein, and Sexl also solved the problem for an ellipsoid, which enabled them to give a solution for a thin circular disc (with the smaller axis of the ellipsoid towards zero). The (Maxwell) creeping flow solution of these authors can be rewritten (with $C_{\mathrm{s}}=3 / 2$ ) as:

$$
F_{\text {Maxwell,Epstein,Sexl }}=6 \pi \frac{\eta^{2} \tilde{R} / \tilde{M}}{p} \frac{\Delta T}{T}
$$

The constant $6 \pi$ for the (thin) circular disc ${ }^{13,14}$ is to be replaced by $3 \pi$ for a spherical particle ${ }^{12,13,14}$. Dividing the force in equation (10) by the surface area of the disc, $L^{2}=(\pi / 4) d^{2}$, and using again the Maxwell velocity, $\bar{w}=[8 \tilde{R} T /(\pi \tilde{M})]^{1 / 2}$, equation (10) can be rewritten in the same form as equation (4):

$$
F_{M, E, S} / L^{2}=\frac{p_{c h}^{2}}{p} \frac{\Delta T}{T} p>>p_{\text {opt }}
$$

and $p_{\mathrm{ch}}$ turns out to be exactly the same as for the tube in equation (5)! By setting the two asymptotic laws of Reynolds (3) and Maxwell (4), and the corresponding laws of Knudsen (6) and Epstein, Sexl (11) equal to each other, the optimal pressures, $p_{\text {opt }}$, are obtained for the tube, or porous slab, as

$$
p_{\text {opt }}=\sqrt{2} p_{c h} \quad \text { (porous slab) }
$$

and for the disc, or vane of a radiometer, as

$$
p_{\text {opt }}=2 p_{c h} \text { (radiometer vane) }
$$

where $p_{\mathrm{ch}}$ is the same value from equation (5), with, $d$, as a mean pore diameter, or the diameter of a circular disc-shaped vane.

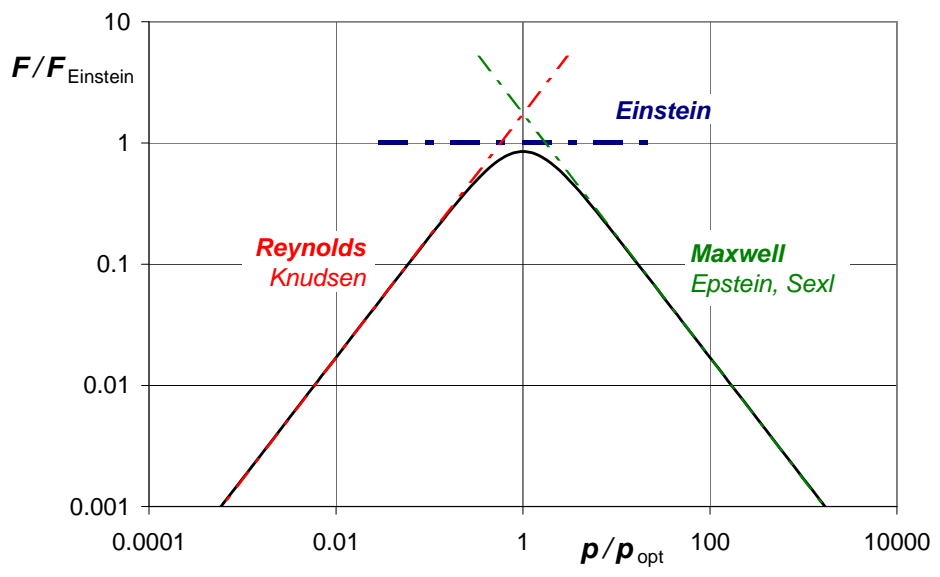

Figure 5 Radiometer forces versus pressure (log-log-plot). Asymptotic solutions for low pressures by Reynolds, and Knudsen, for higher pressures by Maxwell, Epstein, and Sexl. In the range of $p=p_{\text {opt }}$ by Einstein (here: $n=1$, for simplicity).

\section{STRAUB, BRENNER, DURST}

The interest in the scientific explanation of the radiometer forces and related phenomena, which was tremendously great in the early years from 1873 to 1897 , found an interesting second peak in the early 20 ${ }^{\text {th }}$ century, from 1909 to 1930 (see KNUDSEN, WESTPHAL, EINSTEIN). During the second world war, and long after its end, the topic seems to have fallen into oblivion. Only recently, around the beginning of the $21^{\text {st }}$ century, some new applications, like the so-called Knudsen pumps in micro- and submicro-technology, seems to have led to a new wave of interest in these long forgotten effects. The technological aspects, however, though certainly of great practical importance, will not be treated here. It was fascinating to learn that a number of present day scientists have started to develop more generalized sets of thermofluiddynamic equations than those well-known Navier-Stokes, Fourier, and Fick (NSFF) equations, that were looked at as practically sacrocant for centuries. Howard Brenner ${ }^{16,17}$, since 2005, has written a great number of interesting papers dealing with Navier-Stokes, revisited and related topics. Interestingly he started out from phenomena like thermophoresis, and tried to include these into a continuum theory, without the wall-slip boundary condition. This was accomplished by introducing two different velocities into the fluiddynamic equations. Somehow similar attempts were followed by Franz Durst, and his coworkers $^{18,19}$. Earlier, but independently from the attempt to include certain special phenomena into the equations, Dieter Straub ${ }^{15}$ has developed an Alternative Mathematical Theory of Non-equilibrium Phenomena. This much more general theory is based on the so-called Gibbs-Falk dynamics, that is a generalization of Gibbs's ideas about the dependency of energy of a 'system' on appropriately chosen state variables. It may be seen as a kind of unification of thermodynamics with the rest of physics. Unfortunately this theory hasn't found great interest among the majority of present day thermofluiddynamics experts, so far.

\section{CONCLUSION}

The phenomena of thermal transpiration and impulsion, as first investigated and described by Reynolds in 1879, and Maxwell's thermal creeping flow equations, from the same year, which were practically fallen into oblivion among the engineering community, have attracted new interest only recently because of their importance in rarefied gases. Gases at normal pressure have to be treated as rarefied in micro-, and submicro channels. That's why these closely related fundamental physical phenomena ought to be better known to engineering students again, especially so in the fields of thermofluiddynamics. It will be useful to follow the development of our basic equations in the next future. 


\section{ACKNOWLEDGMENTS}

The author wants to thank Mike Scholtes, fellowlimericktionist and workshop editor in OEDILF $^{20}$ (The Omnificent English Dictionary In Limerick Form), who, in May 2009, pointed out that my (earlier) explanation of how a Crookes radiometer works "was discredited a long time ago (before Einstein). It is mentioned in the Wikipedia article on the Crookes radiometer." His comment was the incentive of the literature research that finally led to this conference contribution (and to a more appropriate author's note to my limerick on Crookes radiometer).

\section{REFERENCES}

1 The Kind of Motion We Call Heat, Vol.1, \$5.5 Maxwell, Osborne Reynolds, and the radiometer, Stephen G. Brush.

2 Phil. Trans. Roy. Soc. (London), Vol. 170, pp. 727-845, 1879, XVII. On certain Dimensional Properties of Matter in the Gaseous State, Osborne Reynolds.

3 Phil. Trans. Roy. Soc. (London), Vol. 170, pp. 231-256, 1879, VII. On Stresses in Rarified Gases arising from Inequalities in Temperature, James Clerk Maxwell.

4 Annalen der Physik, Vol. 28, pp. 75-130, 1909, Die Gesetze der Molekularströmung und der inneren Reibungsströmung der Gase durch Röhren, Martin Knudsen

5 Annalen der Physik, Vol. 31, pp. 205-225, 1910a, Eine Revision der Gleichgewichtsbedingung der Gase. Thermische Molekularströmung , Martin Knudsen

6 Annalen der Physik, Vol. 31, pp. 633-640, 1910b, Thermischer Molekulardruck der Gase in Röhren und porösen Körpern, Martin Knudsen

7 Annalen der Physik, Vol. 32, pp. 809-842, 1910c, Ein absolutes Manometer, Martin Knudsen

8 Annalen der Physik, Vol. 388, Nr. 14, pp. 797-821, 1927, Thermischer Molekulardruck in Röhren, Martin Knudsen

9 Annalen der Physik, Vol. 398, Nr. 2, pp. 129-185, 1930, Radiometerdruck und Akkommodationskoeffizient, Martin Knudsen

10 Zeitschrift für Physik, Vol. 1, pp. 92-100, pp. 431-438 (part II), 1920, Vol. 4, pp. 221-225 (part III), 1921, Messungen am Radiometer, Wilhelm $\mathrm{H}$. Westphal

11 Zeitschrift für Physik, Vol. 27, pp. 1-6, 1924, Zur Theorie der Radiometerkräfte, A. Einstein

12 Zeitschrift für Physik, Vol. 37, pp. 179-192, 1926, Zur Theorie der Photophorese, G. Hettner

13 Zeitschrift für Physik, Vol. 52, pp. 249-262, 1928, Zur Theorie des Einplattenradiometers, Theodor Sexl

14 Zeitschrift für Physik, Vol. 54, pp. 537-563, 1929, Zur Theorie des Radiometers, Paul S. Epstein

15 Alternative Mathematical Theory of Non-equilibrium Phenomena, Academic Press, 1996, Dieter Straub
16 Physica A 349, pp. 60-132, 2005, Navier-Stokes revisited, Howard Brenner

17 Physica A 388, pp. 3391-3398, 2009, Bi-velocity hydrodynamics, Howard Brenner

$183^{\text {rd }}$ National $\& 3^{\text {rd }}$ International Conference on Fluid Mechanics and Fluid Power, pp. 1-16, 2005, Extension of the Navier Stokes equations for ideal gas flows with temperature and density gradients, F. Durst, J. Gomez, R. Sambasivan

19 Physics of Fluids, Vol. 19, pp. 088104-1-4, 2007, Derivations of extended Navier-Stokes equations from upscaled molecular transport considerations for compressible ideal gas flows: Towards extended constitutive forms, Suman Chakraborty, Franz Durst

20 OEDILF, The Omnificent English Dictionary in Limerick Form, http://www.oedilf.com ; Chris J. Strolin, (EiC) ...look up: Crookes radiometer in OEDILF! 